\title{
Prevalence of Attention Deficit Hyperactivity Disorder - ADHD among Young Individuals - An Observational Study
}

\author{
Aparna Patel ${ }^{1}$, Jalpa Parikh ${ }^{2}$ \\ ${ }^{1}$ MPT in Neurosciences, Ahmedabad Institute of Medical Sciences \\ ${ }^{2}$ MPT Neurology and Lecturer and PhD Scholar, Ahmedabad Institute of Medical Sciences, \\ Gujarat University, Ahmedabad, India \\ Corresponding Author: Aparna Patel
}

\begin{abstract}
Background: Attention deficit hyperactivity disorder-ADHD is a developmental nervous behavioural disorder mostly diagnosed in childhood and rarely in adults. Characteristic clinical features in adults are mainly, hyperactivity, attention deficit and impulsiveness instability in interpersonal relations and alcohol abuse which can cause poor occupational, academic and social performance in future.

As ADHD is usually undiagnosed in adults because of its poor knowledge of the symptoms. Need of study is to see the prevalence of ADHD among individuals, as ADHD youth are at high risk of a wide range of adverse psychiatric outcomes.
\end{abstract}

Methodology: This observational study was performed in 307 young female \& male of all the fields aged between 18-24, according to Newman's classification. The subjects were screened for ADHD by Adult ADHD SelfReport Scale (ASRS) which has two partsinattentive and hyperactive/impulsive. This questionnaire was sent through Google Form.

Results: Statistical analysis was done using Microsoft Excel 10th version. According to ASR Scale among 307, 5.53\% were highly likely to have inattentiveness and $6.64 \%$ were highly likely to have hyper activeness and impulsiveness. In 207 females 6.28\%, 5.79\% were highly likely to be inattentive, hyperactive/impulsive and in 100 males $9 \%, 4 \%$ were highly likely to be hyperactive/impulsive, inattentive.
Conclusion: Total prevalence of Adult ADHD is $11.40 \%$. Among all the participants, prevalence of ADHD for Part-A (Inattentive) is $5.53 \%$ and Part-B (Hyperactive/Impulsive) is $6.64 \%$. Out of total, $0.977 \%$ got mixed symptoms of ADHD.

Keywords: Young individuals, ASRS, Adult ADHD, Inattentive, Hyperactive, Impulsive

\section{INTRODUCTION}

ADHD is a common neuropsychiatric disorder defined by persistent pattern of inattention and/or hyperactivity/ impulsivity that interferes with functioning or development. ${ }^{1}$ The symptoms are stipulated to be the same for adults as children despite the fact that clinical observations suggest that the frank hyperactivity of childhood ADHD manifests more as a sense of internal restlessness among adults and that adults have more diverse set of deficits than children in higher-level executive functioning and emotional control. ${ }^{2}$

As Attention-deficit/hyperactivity disorder (ADHD) is often perceived as a childhood disorder; however, it affects adults as well. ${ }^{3}$ As adult ADHD was not included in any of the major psychiatric epidemiological surveys that have been carried out around the world since the landmark Epidemiologic Catchment Area study in the early 1980s (Weissman et al, 1996; World Health Organization (WHO) 
International Consortium in Psychiatric Epidemiology, 2000), attempts to estimate adult ADHD prevalence have been based either on extrapolations from childhood prevalence estimates using information from clinical studies regarding the proportion of childhood cases that persist into adulthood or on direct estimates from a small sample. ${ }^{4}$

Attention-deficit hyperactivity disorder is a serious risk factor for comorbid psychiatric disorders (antisocial personality disorder, substance misuse and affective disorders), and also shows significant correlation with poor socio-economic outcome and functional impairment (lower level of education, higher level of unemployment, and higher rates of unsuccessful marriages, criminality and also road traffic accidents). ${ }^{5}$

Adult ADHD is being diagnosed more and more often in the west. Yet in India there is very less research from the adult general population and ADHD is still considered a diagnosable disorder in children only. ${ }^{6}$

\section{Need of the Study}

There is vast literature available on prevalence of ADHD in children. However, there is dearth of information on prevalence of ADHD in young individuals. As ADHD is usually undiagnosed in adults because of its poor knowledge of the symptoms. Need of study is to see the prevalence of ADHD among individuals, as ADHD youth are at high risk of a wide range of adverse psychiatric outcomes including markedly elevated rates of antisocial, addictive, mood, and anxiety and sleep disorder. Also, it goes along with academic underachievement, because of which many of such young individuals fail to reach the college level.

\section{Aim and Objectives}

1. To find prevalence of ADHD among young individuals with Adult ADHD Self-Report Scale

2. To find prevalence of ADHD in Females and Males

\section{MATERIALS AND METHODS Inclusion Criteria}

- Age: 18 -24 according to Newman's classification $^{7}$

- Females and males

- Subjects willing to participate

\section{Exclusion Criteria}

- Already diagnosed with ADHD

- Diagnosed with other psychological disorders

\section{Outcome Measure}

- Adult ADHD self-report scale (ASRS) is an instrument consisting of the eighteen DSM-IV TR criteria. Six of eighteen questions were found to be the most predictive of symptoms consistent with ADHD. These six questions are basis for the ASRS v1.1 Screener and are also Part A of Symptom Checklist. Part B of the Symptom Checklist contains remaining twelve questions. ${ }^{8}$

- Scoring and interpretation: If four or more marks appear in the darkly shaded boxes within Part A then the patient has symptoms highly consistent with ADHD in adults and further investigation is warranted in them. The frequency scores on Part B provide additional cues and can serve as further probes into patient's symptoms. Pay particular attention to marks appearing in dark shaded boxes. The frequency-based response is more sensitive with the certain questions. No total score or diagnostic likelihood is utilized for twelve questions. It has been found that the six questions in Part A are the most predictive of the disorder and are the best for use as a screening instrument.

- $\quad$ Test-retest reliability is High $^{9}$

\section{METHOD}

This questionnaire was sent through internet medium via Google forms. It had two parts where one included the demographic data and inclusion-exclusion criteria's and second part had questioner. The Google form was able to reach 307 
Aparna Patel et.al. Prevalence of attention deficit hyperactivity disorder-ADHD among young individuals-an observational study

people so 307 people could take part in it which included young females \& males of all the fields aged between 18-24, according to the Newman's classification. The scale, Adult ADHD Self-Report Scale (ASRS) has two parts-one is for inattentive and second is for hyperactive/impulsive.

Statistical Analysis: Statistical analysis was done by using Microsoft excel $10^{\text {th }}$ version.

\section{RESULT}

As this questioner was sent through internet medium via Google form, 307 people were able to participate in this study. Out of 307, total females were 207 and males were 100. Average age of 307 people were 21.21 years, where average age for 207 females were 21.16 years and for males 21.30 years.

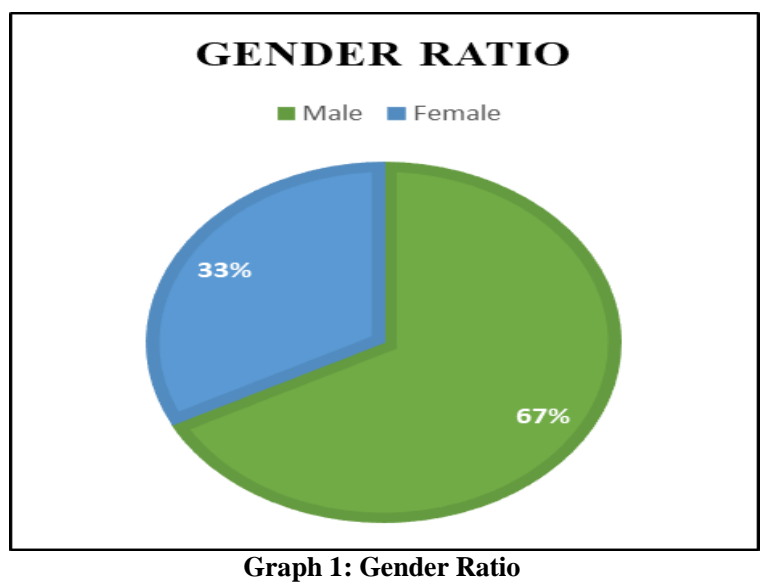

According to ASR Scale among 307, "5.53\%" were highly likely to have inattentiveness and " $6.64 \%$ " were highly likely to have hyper-activeness and impulsiveness.

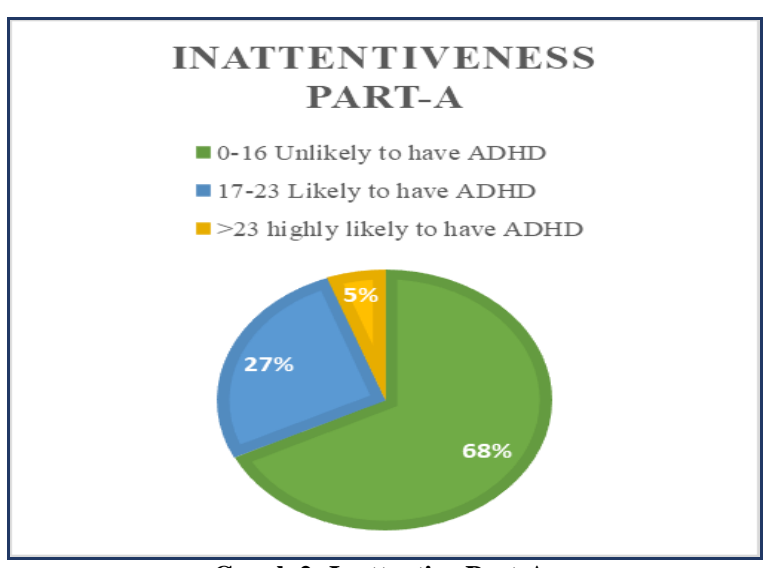

Graph 2: Inattentive Part-A

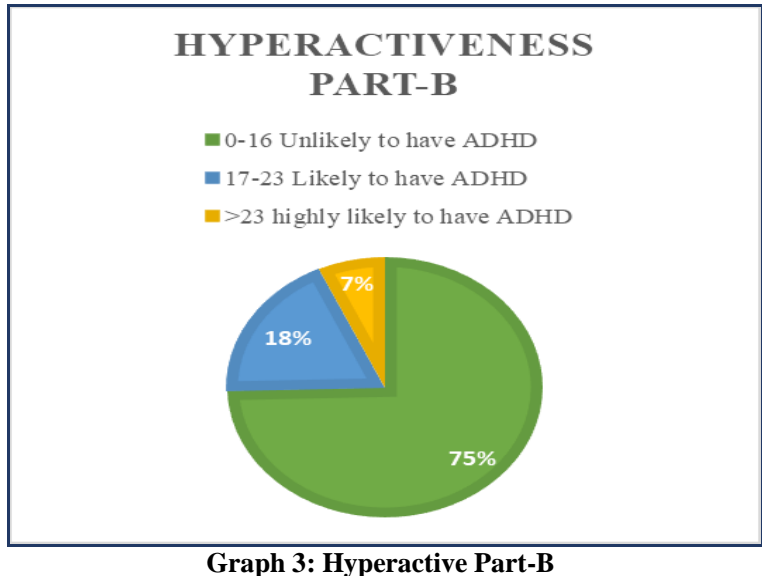

In 207 females "6.28\%" were highly likely to be inattentive and "5.79\%" were highly likely to be hyperactive/impulsive and in 100 males "9\%" were highly likely to be hyperactive/impulsive and $4 \%$ were highly likely to be inattentive. Among total 307 " $0.94 \%$ " people got mixed symptoms of hyperactiveness/impulsiveness and inattentiveness.

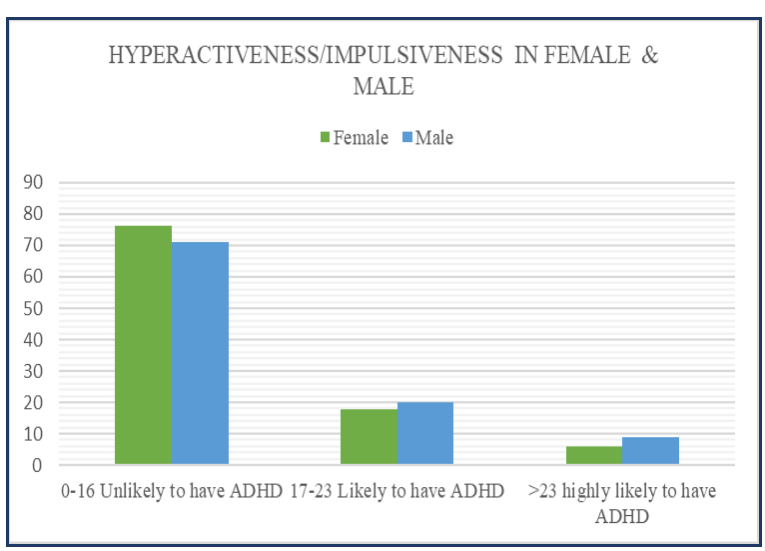

Graph 4: Hyper activeness/Impulsiveness in Female and Male

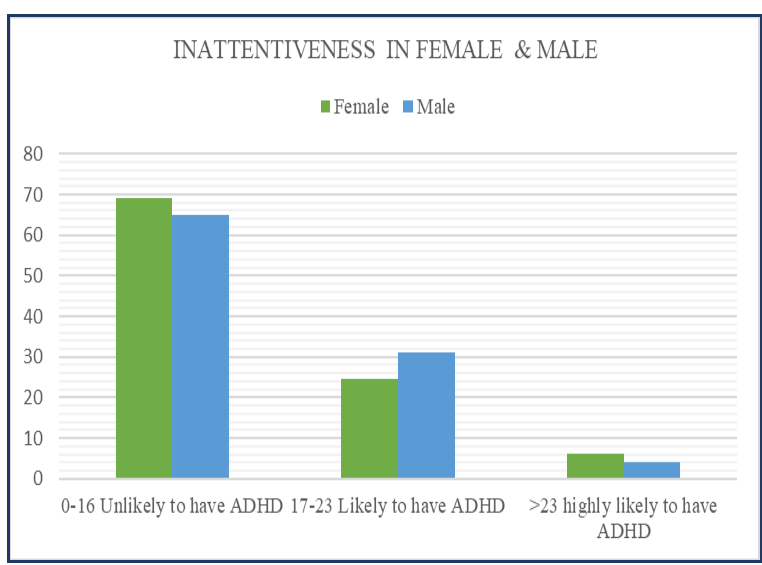

Graph 5: Inattentiveness in Female and Male

Total prevalence of adult ADHD was $11.40 \%$, among total 307 participants. 


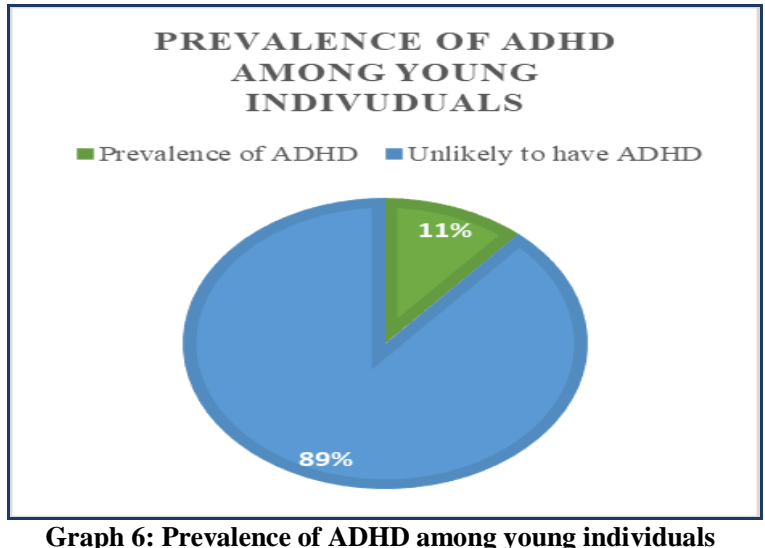

\section{DISCUSSION}

The present study where among all the respondents, $11.40 \%$ were said to be having ADHD. Adult attention-deficit/ hyperactivity disorder (ADHD) is mental health condition, which is exhibited by the difficulty to maintain attention, hyperactivity, and impulsive behavior. The aim of the study was to see the prevalence of ADHD in young adults.

This is supported by an article done by Monire Mosalanejad MD et al., done in 2011 in a study titled "Prevalence of ADHD Among Students of Zahedan University of Medical Science in Iran" concluded that due to high prevalence of ADHD which is seen $15.4 \%$ in the adults, which shows the importance of its early diagnosis and treatment and a need for more frequent screening. ${ }^{10}$

Another prevalence study done by Fernandez et al. in 2006 in a study titled "An estimate of the prevalence of attention deficit hyperactivity disorder (ADHD) among grade one students in Baliwasan central school, Zamboanga City" where 374 students in Baliwasan Central School in Zamboanga participated and has reported an average prevalence of $5.3 \% .^{11}$

Fayyad J. et al. in 2007 in article titled "Cross-national prevalence and correlates of adult attention-deficit hyperactivity disorder" did a similar study in ten countries in the Americas, Europe, and the Middle East on 11,422 respondents aged 18-44 years. Adult ADHD average prevalence was reported to be $3.4 \%$ in the study. ${ }^{12}$
Polanczyk G. et al. in 2007 done a study titled "The worldwide prevalence of ADHD: a systematic review and metaregression analysis" which undertaken a systematic review and stated that worldwide prevalence of ADHD was $5.29 \%$. This suggests that geographic conditions play a limited role in ADHD/HD prevalence estimates worldwide. ${ }^{13}$

Somshekar Reddy Lohit et al. did a study titled "Prevalence of Adult ADHD Co-morbidity in Alcohol Use Disorders in a General Hospital Setup" in 2018 where Adult ADHD was seen in $19 \%$ of patients with AUDs and was associated with rapid progression towards dependence pattern of the use of alcohol. On screening with ASRS, $21 \%(n=21)$ of the subjects were positive for ADHD. After applying a diagnostic interview using DSM 5 in these subjects, ADHD diagnosis was confirmed in $19 \%(\mathrm{n}=19)$ of the subjects. ${ }^{14}$

\section{CONCLUSION}

Total prevalence of Adult ADHD is $11.40 \%$. Among all the participants, prevalence of ADHD for Part-A (Inattentive) is $5.53 \%$ and Part-B (Hyperactive/Impulsive) is $6.64 \%$. Out of total, $0.977 \%$ got mixed symptoms of ADHD.

\section{Clinical Implication}

As ADHD is often misunderstood, many people who have this disorder do not receive appropriate treatment for it and, as a result, may never reach to their full potential. As prevalence is seen in the young adults, regular screening should be done.

\section{Acknowledgement: None}

Conflict of Interest: None

Source of Funding: None

Ethical Approval: Approved

\section{REFERENCES}

1. Instanes JT, Klungsøyr K, Halmøy A, Fasmer OB, Haavik J. Adult ADHD and comorbid somatic disease: a systematic 
literature review. Journal of Attention Disorders. 2018 Feb;22(3):203-28

2. Adler LA, Faraone SV, Spencer TJ, Berglund P, Alperin S, Kessler RC. The structure of adult ADHD. International journal of methods in psychiatric research. 2017 Mar;26(1):e1555.

3. Wolfers T, Arenas AL, Onnink AM, Dammers J, Hoogman M, Zwiers MP, Buitelaar JK, Franke B, Marquand AF, Beckmann CF. Refinement by integration: aggregated effects of multimodal imaging markers on adult ADHD. Journal of psychiatry \& neuroscience: JPN. 2017 Nov; 42(6):386

4. Fayyad J, De Graaf R, Kessler R, Alonso J, Angermeyer $\mathrm{M}$, Demyttenaere $\mathrm{K}$, De Girolamo G, Haro JM, Karam EG, Lara C, Lepine JP. Cross-national prevalence and correlates of adult attention-deficit hyperactivity disorder. The British Journal of Psychiatry. 2007 May;190(5):402-9

5. Simon V, Czobor P, Bálint S, Mészáros A, Bitter I. Prevalence and correlates of adult attention-deficit hyperactivity disorder: meta-analysis. The British Journal of Psychiatry. 2009 Mar;194(3):204-11

6. Jhambh I, Arun P, Garg J. Cross-sectional study of self-reported ADHD symptoms and psychological comorbidity among college students in Chandigarh, India. Industrial psychiatry journal. 2014 Jul;23(2):111

7. Kiatrungrit K, Putthisri S, Hongsanguansri $\mathrm{S}$, Wisajan $\mathrm{P}$, Jullagate $\mathrm{S}$. Validity and reliability of adult ADHD self-report scale Thai version (ASRS-V1. 1 TH). Shanghai archives of psychiatry. 2017 Aug 25;29 (4): 218.

8. Newman BM, Newman PR. Development through life: A psychosocial approach. Cengage Learning; 2017 Apr 12.

9. Silverstein MJ, Alperin S, Faraone SV, Kessler RC, Adler LA. Test-retest reliability of the adult ADHD Self-Report Scale (ASRS) v1. 1 Screener in non-ADHD controls from a primary care physician practice. Family practice. 2018 Jun;35(3): 336-41.

10. Mosalanejad M, Mosalanejad L, Lashkarpour K. Prevalence of ADHD among students of Zahedan University of Medical Science in Iran. Iranian journal of psychiatry and behavioral sciences. 2013;7 (2):83.

11. Instanes JT, Klungsøyr K, Halmøy A, Fasmer OB, Haavik J. Adult ADHD and comorbid somatic disease: a systematic literature review. Journal of Attention Disorders. 2018 Feb;22(3):203-28.

12. Fayyad J, De Graaf R, Kessler R, Alonso J, Angermeyer $\mathrm{M}$, Demyttenaere $\mathrm{K}$, De Girolamo G, Haro JM, Karam EG, Lara C, Lepine JP. Cross-national prevalence and correlates of adult attention-deficit hyperactivity disorder. The British Journal of Psychiatry. 2007 May;190(5):402-9.

13. Polanczyk G, De Lima MS, Horta BL, Biederman J, Rohde LA. The worldwide prevalence of ADHD: a systematic review and metaregression analysis. American journal of psychiatry. 2007 Jun;164(6):9428.

14. Lohit SR, Babu GN, Sharma S, Rao S, Sachin BS, Matkar AV. Prevalence of adult ADHD co-morbidity in alcohol use disorders in a general hospital setup. Indian journal of psychological medicine. 2019 Nov;41(6):523-8.

How to cite this article: Patel A, Parikh J. Prevalence of attention deficit hyperactivity disorder-ADHD among young individuals-an observational study. International Journal of Science \& Healthcare Research. 2021; 6(3): 225-229. DOI: https://doi.org/10.52403/ijshr. 20210739 